\title{
A Proof of the Kikuta-Ruckle Conjecture on Cyclic Caching of Resources
}

\author{
Steve Alpern • Robbert Fokkink • Christos Pelekis
}

Received: 16 May 2011 / Accepted: 1 December 2011 / Published online: 17 December 2011

(C) The Author(s) 2011. This article is published with open access at Springerlink.com

\begin{abstract}
Suppose that a hider possesses a continuously divisible resource that he may distribute around a circle. The resources on a random arc in the circle are lost. The hider has a priori information on the length of the arc and he wants to maximize the probability that the retrieved portion exceeds a critical quantity, which is enough to survive on. We show that there exists an optimal resource distribution, which uses a finite number of point caches of equal size, establishing a conjecture of Kikuta and Ruckle. Our result is related to a conjecture of Samuels' on-tail probabilities.
\end{abstract}

Keywords Resource allocation · Tail probability · Farey numbers

\section{Introduction}

In this paper, we establish a conjecture which arose from work on accumulation games by Kikuta and Ruckle. They considered the problem of how a Hider should distribute a continuously divisible good of mass $y>1$ over a finite number of $N$ locations, to maximize the probability that a critical amount remains, after an antagonistic searcher removes the material from $N-k$ of these locations. This critical

Communicated by Horst Martini.

\section{S. Alpern}

Department of Applied Mathematics, London School of Economics, Houghton Street, London WC2A 2AE, UK

e-mail: s.alpern@1se.ac.uk

R. Fokkink $(\bowtie) \cdot$ C. Pelekis

Institute of Applied Mathematics, TU Delft, P.O. Box 5031, 2600 GA Delft, The Netherlands

e-mail: r.j.fokkink@tudelft.nl

C. Pelekis

e-mail: c.pelekis@tudelft.nl 
amount is normalized to 1 . Kikuta and Ruckle conjecture that, for all parameter values $N, k, y$, there exists an optimal distribution in which all locations with nonzero mass have equal mass. This conjecture, which is related to an old problem of Hoeffding and Shrikhande on maximizing tail probabilities, appears difficult and remains open. Kikuta and Ruckle have studied several variations of their problem [1-4] and the conjecture that we establish in this paper concerns the accumulation game on a circle.

In this paper, we say that a location with nonzero mass is a cache and, instead of accumulation games, we speak of caching games, to emphasize the connection with the problem faced by scatter hoarders, animals like squirrels, who need to have enough resources left to survive the winter. They protect their hoardings against pilferers or natural events, such as floods, by scattering their supply over various caches [5]. A tragic example of failure to retain a critical amount of cached material comes from Robert Scott's 1911 Antarctic expedition. He distributed the oil, needed for cooking and melting snow, into fuel cans to be picked up on the return trip from the pole. Several of these cans were found empty upon return, due to leakage from evaporation through deterioration of the washers. The remaining fuel was not sufficient for survival. Another related tragedy occurred a year later in 1912 with the sinking of the Titanic. The precious resource in this case was air, needed to keep the ship afloat. A modern safety idea at that time was the subdivision of the ship's hull into discrete watertight compartments, i.e., caches storing the precious resource. Fifteen transverse bulkheads created sixteen cells, each of which could be isolated from the adjoining compartment using special doors. ${ }^{1}$ These doors did not always stay intact and the compartments that filled up with water formed a contiguous set, which is exactly the type of caching problem that we consider in this paper.

The paper is organized as follows. In Sect. 2, we exhibit the caching game on a compact metric space and we state our main theorem. In our case, this compact metric space is either the unit circle or a cyclic graph. In Sect. 3, we consider the optimal strategy for the caching game on the unit circle provided the caches are equal. In Sect. 4, we solve the caching game on a cyclic graph. In Sect. 5, we combine the results and prove our main theorem. For parameters $N, k, y$, the optimal size of the cache depends on the rational of smallest denominator in the half-open interval $\left[\frac{k}{N}, \frac{1}{y}\right)$. Such number theoretic implications are quite common for these types of games [6-8].

\section{Caching Games}

The caching game that we define here is what Kikuta and Ruckle call a one-step accumulation game.

Definition 2.1 (Caching game) Let $X$ be a compact metric space and let $\mathcal{F}$ be a family of Borel subsets of $X$. Let $y>1$ be a fixed real number. The hoarder $H$ chooses a Borel measure $\mu$ on $X$ such that $\mu(X)=y$. The pilferer $P$ chooses a subset $F \in \mathcal{F}$. $H$ wins iff $\mu(F) \geq 1$.

\footnotetext{
${ }^{1}$ http://www.titanic-titanic.com/titanic_watertight_compartments.shtml.
} 
This is a zero-sum, win-lose game in which the payoff to the hoarder is 1 or 0 iff he wins or loses. The measure $\mu$ represents the way that the hoarder distributes the material over the space $X$. The set in $\mathcal{F}$ represents the part of $X$ that is not being pilfered. Our caching game is related to a win-lose game that has been studied in [9]: $P$ chooses a closed subset $F$ of bounded diameter in $X$ and $H$ chooses a point $x$. Then $H$ wins iff $x \in F$. In other words, $H$ chooses a point measure $\mu_{x}$ and wins iff $\mu_{x}(F)>0$. Another related game is the hypergraph incidence game in which $X$ is finite and $H$ again chooses a point [10, p. 5].

If $X$ is finite, then we have a finite game with a well-defined value. For infinite $X$, some conditions on $\mathcal{F}$ are necessary to ensure the existence of the value. By the minimax theorem in [11, Appendix A], the game has a value if $\mathcal{F}$ admits the structure of a compact metric space such that the function $F \mapsto \mu(F)$ is upper semicontinuous. If $\mathcal{F}_{\delta}$ is the family of closed sets (or open sets) in $X$ of diameter $\leq \delta$, then it is compact under the Hausdorff metric and $F \mapsto \mu(F)$ is upper semi-continuous. Similarly, if $\mathcal{F}_{x}$ is the family of closed sets (or open sets) of Lebesgue measure $\leq x$ on the unit interval, then it is compact under the Hausdorff metric. In all these cases, the value of the game is well defined and it can be thought of as the probability that the hoarder wins with best play on both sides. If $X$ is finite, then we have a game on a hypergraph with node set $X$ and edge set $\mathcal{F}$. We say that such a caching game is discrete. If $X$ is infinite, then we say that the game is continuous. In this paper, we will consider a continuous game on the circle and a discrete game on the cyclic graph. These games are intimately related.

\subsection{The Kikuta-Ruckle Conjectures}

Kikuta and Ruckle have conjectured for several caching games that an optimal $\mu$ consists of finitely many atoms that have equal weight. Or more succinctly, they conjectured that $\mu$ has equal caches. Their conjectures [3, Conjecture 11] and [2, Conjecture 3.1] apply to the following games:

(1) $X=\{1, \ldots, N\}$ is finite and $\mathcal{F}$ consists of all subsets of $X$ of a fixed cardinality $k<N$. This is the caching game that we describe at the beginning of the paper.

(2) $X=\{1, \ldots, N\}$ is finite and $\mathcal{F}$ consists of all subsets of $X$ that contain $k$ consecutive numbers modulo $N$. This is a caching game on a cyclic graph.

(3) $X$ is the circle of unit circumference and $\mathcal{F}$ consists of all closed intervals of fixed length $x$.

For all these games, it is intuitively clear that the pilferer selects a subset of $\mathcal{F}_{k}$ randomly and equiprobable. A proof of this can be found in [3]. What is then required to solve the game, with $P$ 's strategy known, is to determine $H$ 's optimal strategy, and this is an optimization problem. We will solve that problem for the second and the third caching game. Actually, we will see below that the second game is a special case of the third game. We will also provide a partial solution of the first caching game that extends previous results in $[12,13]$.

It turns out that in the third game, it is more convenient to work with half-open intervals $[a, a+x)$ instead of closed intervals. It is not hard to apply the solution of this caching game with half-open intervals to the games in which intervals are either closed or open. We will show how to do that at the end of the paper. 


\subsection{The Hoeffding-Shrikhande Problem}

It is clear that the game on the cyclic graph and the game on the circle are similar. More precisely, consider the game on the cyclic graph of cardinality $N$ with intervals of $k$ consecutive elements and with a measure $\mu$ of weight $y$. Keep the weight $y$ and the ratio $k / N$ fixed while letting $N$ go to infinity. Then in the limit one obtains the caching game on the circle.

Instead of keeping $k / N$ and $y$ fixed while letting $N$ go to infinity, it is natural to consider keeping $y / N$ and $k$ fixed. This does not lead to a caching game but it leads to a well-known problem in probability. Since $P$ samples $k$ locations, we get $k$ random variables $X_{1}, \ldots, X_{k}$ that are identically distributed and of expected value $\bar{y}$. The hoarder wants to maximize $\mathbb{P}\left(X_{1}+\cdots+X_{k} \geq 1\right)$. If these random variables are independent, then we arrive at the to maximize the tail probability $\mathbb{P}\left(X_{1}+\cdots+X_{k} \geq 1\right)$ for i.i.d. nonnegative random variables $X_{i}$ of fixed expectation $\bar{y}$. This is a wellknown problem that goes back to Hoeffding and Shrikhande [14]. It is known [15] that the tail probability is maximized by random variables that assume only two values, i.e., either $Y_{i}=a$ or $Y_{i}=b$ for some nonnegative $a \leq b$. Determining the optimal $a$ and $b$ is a notorious problem. It has been widely conjectured that one may take $a=0$, see [16]. This is analogous to the Kikuta-Ruckle conjecture that all locations are either empty or have a cache of equal size. We briefly discuss this tail probability problem in the final section.

\subsection{Notation and Statement of the Main Theorem}

From now on, $x$ denotes the length of the family of half-open intervals $\mathcal{F}$ in the circle caching game. Note that the circle caching game is trivial if $x y \geq 1$, when $H$ wins for sure by taking $\mu$ as the uniform measure with constant density $y$. So we assume henceforth that $x y<1$.

It turns out that the solution of the circle caching game involves Farey sequences. Recall that the Farey sequence of order $v$ is the increasing sequence of fractions between 0 and 1 which, when in lowest terms, have denominators less than or equal to $v$. For example the Farey sequence of order 5 is given by

$$
\frac{0}{1}, \frac{1}{5}, \frac{1}{4}, \frac{1}{3}, \frac{2}{5}, \frac{1}{2}, \frac{3}{5}, \frac{2}{3}, \frac{3}{4}, \frac{4}{5}, \frac{1}{1} .
$$

Definition 2.2 Given the quantity parameters $x y<1$ from the circle caching game, we define two reduced fractions $\frac{p}{q}$, called the main fraction, and $\frac{r}{s}$, called the successor fraction, as follows:

$p / q$ is the unique rational number of smallest denominator in $[x, 1 / y)$

$r / s$ is the successor of $p / q$ in the Farey sequence of order $q$.

We note that the uniqueness of $\frac{p}{q}$ follows from the well-known fact that adjacent fractions in a Farey sequence have different denominators. Applying this to the Farey sequence of order $q$, we conclude that between fractions of denominator $q$ there is an intervening fraction of smaller denominator. 
Fig. 1 For $x, y$ in the shaded region, the hoarder places unit caches in the circle caching game

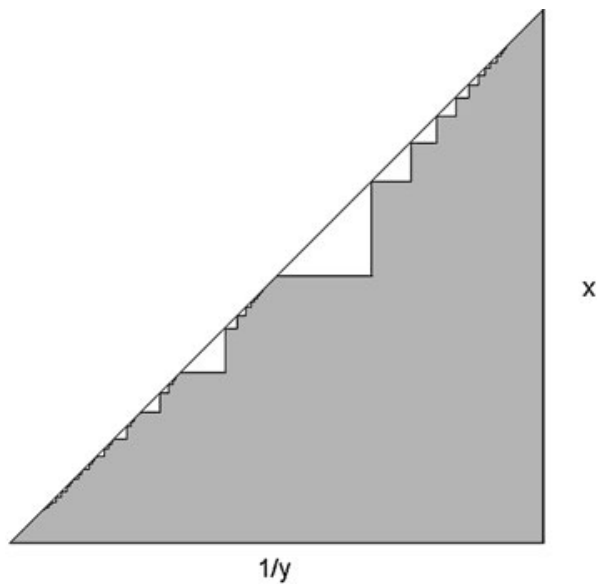

Theorem 2.1 In the circle caching game with parameters $x$ and $y$, and successor fraction $r / s$, there is an optimal $\mu$ consisting of caches of weight $1 / r$ placed at the $s$ points $i x / r, i=1,2, \ldots, s$. The value of the game is $1+s x-r$. In particular, the Kikuta-Ruckle conjecture on equal caching holds for this game.

We will prove this theorem, which is our main result, in the course of the paper. For many values of $x, y$, the successor fraction has numerator 1 , in which case the hoarder places unit caches. The region of such $x, y$ is shaded in Fig. 1. The hoarder places smaller caches only if the gap between $x$ and $\frac{1}{y}$ is small. The shaded region is bounded by infinite staircases: if $j$ is an integer and $\frac{1}{j+1} \leq \frac{1}{y} \leq \frac{1}{j}$, then the top of the shaded region is a staircase that starts at $\frac{1}{j+1}$ and as $\frac{1}{y}$ increases it runs up to $\frac{1}{j}$ through the steps at $\frac{i}{i j+1}$ for $i=1,2, \ldots$.

\section{Optimizing $\mu$ that Have Equidistant Equal Caches}

In this section, we assume that $\mu$ has equal caches and determine the optimal size of the cache. Since $\mu$ represents a division of goods over the circle, we say that it is an allocation. Clearly, if $\mu$ is an allocation with equal caches, then $\mu(I) \geq 1$ if and only if the interval $I$ contains enough caches, so we may restrict our attention to caches of size $\frac{1}{n}$ for integers $n \geq 1$. We say that an interval is heavy if it contains at least $n$ caches, otherwise it is light.

We define the allocation $\mu_{n}$ as the one that has as many caches of size $\frac{1}{n}$ as possible at the locations $0, x / n, 2 x / n, \ldots,(m-1) x / n$. Note that $\frac{m}{n}$ is the largest rational of denominator $n$ that is $\leq y$. The gap between the caches is equal to $x / n$, except for the final gap between $(m-1) x / n$ and 0 . We denote $\delta=x / n$ and we denote the final gap by $\Delta$. Note that any interval $[t, t+x)$ contains at most $n$ caches and that $m \delta<1$. Also note that $\Delta>\delta$ and that an interval $[t, t+x)$ that does not intersect the final gap is heavy.

Now that we have fixed $\delta$ dependent on $n$, we optimize the probability $\operatorname{Pr}_{n}$ that a random interval $[t, t+x)$ is heavy. More specifically, if $L_{n}=\left\{t: \mu_{n}[t, t+x) \geq 1\right\}$, 
then the probability that a random interval is heavy is equal to $\operatorname{Pr}_{n}=\lambda\left(L_{n}\right)$, the Lebesgue measure of $L_{n}$.

Lemma 3.1 If $n$ is such that $\Delta \geq 2 \delta$, then $\operatorname{Pr}_{n} \leq \operatorname{Pr}_{1}$.

Proof We claim that $L_{n}^{c}=(1-x-\Delta+\delta, 1-\delta]$, which implies that $\operatorname{Pr}_{n}$ equals $x+\Delta-2 \delta=1+x-\frac{(m+1) x}{n}$. In other words,

$$
\operatorname{Pr}_{n}=x\left(\frac{m+1}{n}-1\right) .
$$

To maximize this probability, we need to maximize $\frac{m+1}{n}$ under the restriction that $\frac{m}{n} \leq y$. If $n=1$ then $\frac{m+1}{n}$ is equal to $\lfloor y\rfloor+1$. Now note that $y<\lfloor y\rfloor+1$, so that $\frac{m}{n} \leq\lfloor y\rfloor+1-\frac{1}{n}$ and we conclude that $\frac{m+1}{n} \leq\lfloor y\rfloor+1$. Thus, it follows from the claim that $P r_{n}$ is maximized at $n=1$ if $\Delta \geq 2 \delta$.

To prove the claim, take an interval $[t, t+x)$ and let $t$ increase from 0 to 1 . The caches are spaced in such a way that every time the interval loses a cache at $t$ it picks up a new cache at $t+x$, until $t+x$ is equal to $1-\Delta+\delta$. In other words, if $t=1-x-\Delta+\delta$ is the infimum of $L_{n}^{c}$. As $t$ increases further, the interval drops another cache once $t+x$ is equal to $1-\Delta+2 \delta$ before it picks up the cache at 0 . The interval regains $n$ caches only once $t>1-\delta$.

Lemma 3.2 To maximize $\operatorname{Pr}_{n}$ we need to maximize $1+m x-n$ under the constraint that $\frac{n}{m} \geq \frac{1}{y}$.

Proof If $\Delta \geq 2 \delta$ then it is optimal to choose $n=1$. In this case, an interval $[t, t+x)$ contains either zero or one cache. We claim that if $\Delta<2 \delta$ then each interval contains either $n$ or $n-1$ caches. To see this, notice that if we place just one extra cache at $1-\Delta+\delta$ then the gaps between all the caches are $\leq \delta$ and all intervals are heavy. So, $\operatorname{Pr}_{n}$ is maximized by an allocation $\mu_{n, \delta}$ such that all intervals contain either $n-1$ or $n$ caches. The expected number of caches in a random interval of length $x$ is $m x$. In other words, $m x=n \operatorname{Pr}_{n}+(n-1)\left(1-P r_{n}\right)$ and we find that $\operatorname{Pr}_{n}=1+m x-n$. We need to maximize this probability under the constraint that $\frac{m}{n} \leq y$.

The optimization of $1+m x-n$ is an exercise in Diophantine approximation, for which we shall need some standard facts concerning Farey sequences that can be found in $[17$, p. 28].

Lemma 3.3 The fraction $\frac{n}{m}$ that maximizes $1+m x-n$ is the successor fraction $\frac{r}{s}$.

Proof We denote the main fraction $\frac{p}{q}$ by $z$. For a rational $\frac{n}{m}>z$, we calculate that $1+$ $m z-n=1+\frac{p m-q n}{q} \geq 1-\frac{1}{q}$. Since $p s-q r=-1$ for the Farey successor and no other rational in $\mathcal{F}_{q}$ has this property, we conclude that $\frac{r}{s}$ is the rational of minimal denominator that maximizes $1+m z-n$. Now suppose that there exists rational $\frac{n}{m}$ such that $1+m x-n>1+s x-r$. By the property of the Farey successor $1+m z-$ $n \leq 1+s z-r$ and by subtracting the first inequality from the second we get $m(z-$ 
$x)<s(z-x)$, which implies that $m<s$. Hence, $\frac{n}{m} \in \mathcal{F}_{q}$ and since $\frac{r}{s}$ is the Farey successor, it follows that $\frac{n}{m}>\frac{r}{s}$. The first inequality is equivalent to $x<\frac{r-n}{s-m}$ and the second inequality is equivalent to $z \geq \frac{r-n}{s-m}$. Now notice that $s-m<s<q$, so this implies that $\frac{r-n}{s-m}$ is a rational in $\left[x, \frac{1}{y}\right)$ of denominator $<q$, contradicting our choice of $\frac{p}{q}$.

Thus, we have proved that the allocation $\mu_{r, \delta}$ maximizes the probability that a random interval is heavy, if $\delta=\frac{x}{r}$. There are other allocations that maximize this probability. Indeed, in the next section we construct another optimal allocation which has caches that are not equidistant.

\section{Solution of the Circle Caching Game for Rational $x$}

In this section, we solve the circle caching game for rational $x=\frac{a}{b}$. It turns out that it is equivalent to the cyclic graph caching game.

Lemma 4.1 The circle caching game for rational $x=\frac{a}{b}$ is equivalent to the caching game on the cyclic graph with $b$ nodes and discrete intervals of length $a$.

Proof Place the $b$ nodes of the cyclic graph on the unit circle at the positions $\frac{j}{b}$ for integer $0 \leq j<b$. Note that any interval $[t, t+x)$ intersects exactly $a$ consecutive nodes, so the intersection of the family $\mathcal{F}$ of half-open intervals in the circle caching game gives the family of consecutive nodes in the caching game on the cyclic graph. Observe that any allocation in the caching game on the cyclic graph can be used in the circle caching game, by allocating the material to the points $\frac{j}{b}$ in the circle. Therefore, the circle caching game is at least as good for the hider as the cyclic graph caching game.

Suppose that the pilferer only selects intervals $\left[\frac{i}{b}, \frac{i}{b}+x\right)$ in the circle caching game. The weight of such an interval is equal to the sum of all $\mu\left[\frac{j}{b}, \frac{j+1}{b}\right)$ for all the points $\frac{j}{b}$ that are in the interval. So, if the pilferer only selects these intervals, then the hider may just as well allocate the material to the points $\frac{j}{b}$ only. In other words, by selecting these intervals $P$ forces $H$ to play the cyclic caching game. Therefore, the circle graph caching game with $x=\frac{a}{b}$ is at least as good for the pilferer as the cyclic caching game on $b$ nodes with intervals of length $a$. We conclude that the two games are equivalent.

Note that we may choose any $a$ and $b$ such that $x=\frac{a}{b}$. In particular, we may assume that $a$ and $b$ are coprime. We solve the cyclic graph game on $b$ nodes and discrete intervals of length $a$. It is convenient to do our arithmetic modulo $b$ and represent the nodes by $\mathbb{Z} / b \mathbb{Z}$. We denote a discrete interval subset of $a$ nodes by $I_{j}=$ $[j, j+a-1] \subset \mathbb{Z} / b \mathbb{Z}$. The problem now is to determine the optimal allocation $\mu$ that maximizes the number of heavy intervals $I_{j}$.

Lemma 4.2 Let $\mu$ be an optimal allocation with a minimum number of caches such one cache has weight 1 . Then all caches are 1 . 
Proof Suppose that $\mu(0) \geq 1$. Then all intervals that contain 0 are heavy. Hence, we have to distribute the remaining material, which has total weight $y-1$, in such a way that the number of heavy intervals that do not contain 0 is maximal. So, we have to solve the caching game for $y-1$ on the set $\{0, \ldots, b-1\}$ with $\mathcal{F}$ the family of sets of $a$ consecutive nodes and now $b-1$ and 0 are not consecutive. This is the caching game on the linear graph, for which Kikuta and Ruckle have showed in [3, Theorem 26] that is optimal to use unit caches.

Lemma 4.3 If $\mu$ is an optimal allocation with a minimum number of caches, then the end points of all light intervals have zero mass.

Proof We argue by contradiction. Suppose that $I_{j}$ is light and that $\mu\{j\}>0$. Choose the minimal $k \geq 1$ such that $\mu\{j-k\}>0$. Transfer the mass from $j$ to $j-k$ to get a new allocation $\tilde{\mu}$. This transfer reduces the number of caches by one. We claim that no interval that is heavy under $\mu$ can be light under $\tilde{\mu}$. To see this, note that $\tilde{\mu}\left(I_{i}\right)<\mu\left(I_{i}\right)$ if and only if $j \in I_{i}$ and $j-k \notin I_{i}$. This holds only for $i \in\{j-k+1, \ldots, j\}$, but only one of these nodes has a cache under $\mu$, namely node $j$. It follows that $\mu\left(I_{i}\right) \leq$ $\mu\left(I_{j}\right)<1$ for $i \in\{j-k+1, \ldots, j\}$, so all intervals that get a reduced mass under $\tilde{\mu}$ were light under $\mu$ anyway. It follows that $\tilde{\mu}$ remains optimal. This contradicts our choice that $\mu$ has a minimal number of caches, hence light intervals have end points of mass zero.

We introduce the function $\zeta(j)$ that counts the number of caches in $I_{j}$ under an allocation $\mu$.

\section{Lemma 4.4 There exist an optimal allocation such that:}

(i) $\zeta(j)=n-1$ if $I_{j}$ is light and $\zeta(j)=n$ if $I_{j}$ is heavy, for a fixed number $n$;

(ii) Any $n$ consecutive caches weigh $\geq 1$.

Proof Our standing assumption is that $\mu$ is an optimal allocation with a minimum number of caches. By the previous lemma, we may assume that end points of light intervals have mass zero. It follows that $\zeta(j)=\zeta(j+1)$ if both $I_{j}$ and $I_{j+1}$ are light and that $\zeta(j)+1=\zeta(j+1)$ if $I_{j}$ is light and $I_{j+1}$ is heavy. If there is a light interval without any caches, then by Lemma 4.2 all caches are unit caches. So, we may assume that there are no $a-1$ consecutive locations without any cache.

Suppose that $I_{j}$ is light for $j=1, \ldots, k$ and that $I_{k+1}$ is heavy. Then the locations $a, \ldots, a+k-1$ are empty and there is a cache at $a+k$. Transfer the cache from $a+k$ to $a$. Then the intervals $I_{j}$ for $j=1, \ldots, k$ become heavy, since they all contain the location $a$. On the other hand, the $k$ intervals $I_{j}$ for $j=a+1, \ldots, a+k$ reduce in weight. Since $\mu$ is optimal and since $k$ light intervals became heavy, all these intervals $I_{j}$ for $j=a+1, \ldots, a+k$ were heavy and become light. Before the transfer, the index set $J=\{1, \ldots, k\}$ corresponded to light intervals. After the transfer, the index set $a+J$ corresponds to light intervals. The intervals $I_{i}$ with $i \notin J \cup a+J$ are not affected by the transfer. They remain heavy or light.

Consider the index set of light intervals $L=\left\{j: \mu\left(I_{j}\right)<1\right\}$. We have seen that by a single transfer, a maximal subinterval $J \subset L$ is rotated to $a+J$ and all other indices 
are unaffected. Since $a$ and $b$ are coprime, there exists an iterate of the rotation $j \mapsto$ $a+j$ that is equal to the rotation by one. Therefore, we can rotate any maximal interval in $L$ by one. It follows that we can apply a finite number of cache transfers such that $L$ consists of one interval only. In other words, we may assume that all light intervals are consecutive. Since $\zeta$ is constant on $L$, all light intervals contain the same number of caches. Furthermore, we can rotate $L$ over any multiple of $a$. Each time we apply the transfer, heavy intervals become light by losing just one cache. It follows that all heavy intervals can be made light by removing just a single cache. So, $\zeta$ is constant on the heavy intervals as well. This takes care of statement (i).

To prove statement (ii), we may assume that $L=\{1, \ldots, k\}$. We know that $\zeta(j)=$ $n-1$ for all $j \in L$ and $\zeta(j)=n$ for all $j \notin L$. By Lemma 4.3, we may assume that $L$ has no cache, so all $I_{j}$ with $j \in L$ contain the same $n-1$ consecutive caches. Since $I_{0}$ is heavy and $I_{1}$ is not, there is a cache at 0 that is previous to the $n-1$ caches in the light intervals. Similarly, since $I_{k+1}$ is heavy and $I_{k}$ is not, there is a cache at $a+k$ that is consecutive to the $n-1$ caches in the light intervals. It follows that any $n$ consecutive caches are contained in a heavy interval.

Theorem 4.1 Consider the caching game on the unit circle with $\mathcal{F}$ the family of half open intervals $[r, r+x)$ of fixed rational length $x$. Let $r / s$ be the successor fraction. There exists an optimal allocation with s equidistant caches of size $\frac{1}{r}$ and the value of the game is $1+s x-r$.

Proof By Lemma 4.1, this game is equivalent to the caching game on the cyclic graph. By the previous lemma, there exists an optimal allocation on the cyclic graph such that a heavy interval contains $n$ caches while a light interval contains $n-1$ caches. Let $d$ be the number of heavy intervals; $m$ is the number of caches; and $x=a / b$. The expected number of caches in a random interval of length $a$ is $a m / b$. There are $d$ intervals that contain $n$ caches. The remaining $n-d$ light intervals each contain $n-1$ caches. So,

$$
a m / b=n d / b+(n-1)(b-d) / b \quad \Longrightarrow \quad 1+m x-n=d / b .
$$

Note that $d / b$ is the probability of a win for the hoarder. Also note that $m / n \leq y$, since there are $m$ caches of weight $1 / n$. So, we reencounter the constraint that the value of the game is $\leq 1+m x-n$ for some $m / n \leq y$. The maximal value of $1+m x-n$ is attained by the successor fraction and we know from our analysis of equidistant allocations that this value is attainable.

\section{Solution of the Circle Caching Game for Arbitrary $x$}

We have solved the circle caching game for rational $x$. It is not difficult to extend the solution to general $x$.

Proof of Theorem 2.1 For rational $x$, a random half-open interval of length $x$ is heavy with probability $1+r x-s$, where $r / s$ is the Farey successor. For an optimal allocation $\mu$, a random half-open interval of length $x$ is heavy with a probability that 
depends on $x$ and $y$. We denote this probability by $\operatorname{Pr}(x, y)$. Obviously, $\operatorname{Pr}(x, y)$ is nondecreasing in $x$ and $y$. For a fixed $y$, the rational of minimal denominator $\frac{p}{q}$ remains the same if $x$ increases as long as $x \leq \frac{p}{q}$. Therefore, the value of the game does not change if $x$ increases, as long as $x$ is rational and $x \leq \frac{p}{q}$. Since $\operatorname{Pr}(x, y)$ is nondecreasing in $x$, this also applies to nonrational $x$ by monotonicity.

Corollary 5.1 Consider the circle caching game for which $\mathcal{F}$ is the family of with closed intervals of length $x$. The value of the game is probability is $1+r x-s$ where $r / s$ is the Farey successor of the rational $p / q \in\left(x, \frac{1}{y}\right)$ of smallest denominator.

Proof Let $\operatorname{Pr}[x, y]$ be the probability that a closed interval of length $x$ is heavy, for an allocation $\mu$ of weight $y$. Similarly, $\operatorname{Pr}(x, y)$ is the probability that an open interval of length $x$ is heavy. We assume that $\mu$ is optimal in the circle caching game with closed intervals. Then $\operatorname{Pr}(x, y) \leq \operatorname{Pr}[x, y] \leq \operatorname{Pr}(x+\epsilon, y)$ for all $\epsilon>0$. Now $\lim _{\epsilon \rightarrow 0} \operatorname{Pr}(x+\epsilon, y)=\operatorname{Pr}(x, y)$ unless $x$ is equal to the rational of smallest denominator in $[x, 1 / y)$. For such an $x, \lim _{\epsilon \rightarrow 0} \operatorname{Pr}(x+\epsilon, y)$ is equal to $1+r^{\prime} x-s^{\prime}$ where $r^{\prime} / s^{\prime}$ is equal to the Farey successor of the rational of minimal denominator $p^{\prime} / q^{\prime} \in(x, 1 / y)$. To see that $\operatorname{Pr}[x, y]$ is actually equal to the limit of $\operatorname{Pr}(x+\epsilon, y)$, use that $\lim _{\epsilon \rightarrow 0} \mu[a, x+\epsilon)=\mu[a, x]$.

As an application of our results, we can settle an instance of the Kikuta-Ruckle conjecture on the discrete caching game in which $\mathcal{F}$ is the family of subsets of a fixed cardinality.

Corollary 5.2 Consider the discrete caching game in which $X$ is a set of $N$ elements and $\mathcal{F}_{k}$ consists of all subsets of $k$ elements of $X$. Suppose that $x=k / N, y$ are such that $\frac{j-1}{j} \in[x, 1 / y)$ for some $j$. Then it is optimal for the hider to use a single cache of unit weight.

Proof First observe that the family $\mathcal{F}$ for the cyclic caching game is a subset of the family $\mathcal{F}_{k}$ of all subsets of $k$ elements. So, the value of the cyclic caching game is less than or equal to the value of the caching game with $\mathcal{F}_{k}$. For the given parameter values, the Farey successor is equal to $1 / 1$. So, the optimal allocation for the cyclic caching game consists of one unit cache. The probability that a random element of $\mathcal{F}$ contains the cache is equal to $x$. The probability that a random element of $\mathcal{F}_{k}$ contains the cache is the same. So, both caching games have the same value for these parameter values.

The result in Corollary 5.2 is illustrated in Fig. 2 below.

\section{Concluding Remarks}

Our solution of the circle caching game shows a certain monotonicity. If we decrease $x$ while keeping $y$ fixed, then the numerator $r$ of the Farey successor decreases. 
Fig. 2 The shaded region represents the parameters $x, y$ that are easy-in this case. The hoarder places one unit cache in the discrete caching game

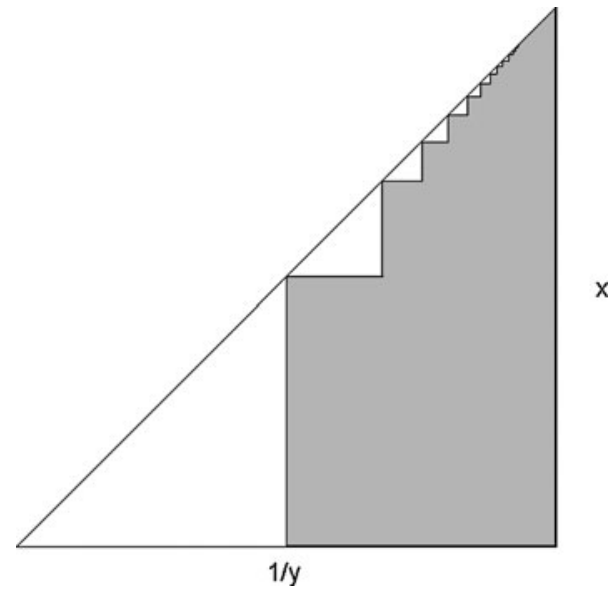

In other words, if the size of the gap between $x$ and $\frac{1}{y}$ decreases, then the size of the cache increases. One would expect to find the same monotonicity in the solution of the discrete caching game, if the Kikuta-Ruckle conjecture turns out to be true. A similar monotonicity has been observed by Samuels [15]. It is already apparent in the theorem of Hoeffding-Shrikhande:

Theorem 6.1 The tail probability $\mathbb{P}\left(X_{1}+X_{2} \geq 1\right)$ of a sum of two i.i.d. non-negative random variables, under the constraint that $\mathbb{E}\left[X_{i}\right]=\bar{y}$ for $i=1,2$, is maximized by random variables that take at most two values. If $\frac{2}{5} \leq \bar{y}<\frac{1}{2}$, the probability is maximized by $X_{i}$ that take the values 0 and $\frac{1}{2}$. If $\bar{y}<\frac{2}{5}$, the probability is maximized by $X_{i}$ that take the values 0 and 1 .

In other words, if $\bar{y}$ decreases, then the positive value of the random variable (the cache) increases. Samuels proved that the Hoeffding-Shrikhande problem is solved by random variables $Y_{i}$ such that $Y_{i}=0$ or $Y_{i}=1$ if $k \bar{y} \leq \min \left\{\frac{1}{k-1}, \frac{1}{4}\right\}$. In a discussion at the end of that paper, Samuels includes a conjecture on the solution of the Hoeffding-Shrikhande problem that more or less comes down to the following.

Conjecture 6.1 Suppose that the tail probability $\mathbb{P}\left(X_{1}+\cdots+X_{k} \geq 1\right)$ of a sum of $k$ i.i.d. nonnegative random variables, under the constraint that $\mathbb{E}\left[X_{i}\right]=\bar{y}$, be maximized by random variables that take the values 0 and 1 . Then this remains true if we decrease $k$ or if we decrease $\bar{y}$.

We can incorporate a similar monotonicity in the Kikuta-Ruckle conjecture, as follows.

Conjecture 6.2 (Generalized Kikuta-Ruckle) Consider a caching game on a compact metric space $X$. The pilferer may choose an element of a family $\mathcal{F}$ and the hoarder may choose a measure $\mu$ total mass $y$. If there exists a transformation group of $X$ that acts transitively on $\mathcal{F}$, then there exists an optimal $\mu$ that has equal caches. The size of the caches increases if $y$ decreases. 
We have solved this conjecture in case $X$ is the circle group and $\mathcal{F}$ is a family of intervals. As a next step, it is natural to consider a homogeneous space $X$ with a transitive transformation group $G$ and a family $\mathcal{F}$ that consists of translates $G \cdot V$ of a single subset $V \subset X$. For instance, $X$ is a torus and $V$ is a rectangle. This caching game on the torus has a flavor of covering problems in combinatorial geometry.

Open Access This article is distributed under the terms of the Creative Commons Attribution Noncommercial License which permits any noncommercial use, distribution, and reproduction in any medium, provided the original author(s) and source are credited.

\section{References}

1. Kikuta, K., Ruckle, W.: Accumulation games, part 1: noisy search. J. Optim. Theory Appl. 94(2), 395-408 (1997)

2. Kikuta, K., Ruckle, W.: Continuous accumulation games in continuous regions. J. Optim. Theory Appl. 106(3), 581-601 (2000)

3. Kikuta, K., Ruckle, W.: Continuous accumulation games on discrete locations. Nav. Res. Logist. 41(6), 821-831 (2001)

4. Ruckle, W.: Accumulation games. Sci. Math. Jpn. 54(1), 173-203 (2001)

5. Morris, D.: The behaviour of the green acouchi (Myoprocta pratti) with special reference to scatter hoarding. Proc. Zool. Soc. Lond. 139, 701-732 (1962)

6. Baston, V., Bostock, F.A., Ferguson, T.S.: The number hides game. Proc. Am. Math. Soc. 107(2), 437-447 (1989)

7. Baston, V., Kikuta, K.: An ambush game with a fat infiltrator. Oper. Res. 57(2), 514-519 (2009)

8. Zoroa, N., Fernandez-Saez, M.J., Zoroa, P.: A game related to the number of hides game. J. Optim. Theory Appl. 107(2), 457-473 (1999)

9. McEliece, R., Posner, E.C.: Hiding and covering in a compact metric space. Ann. Stat. 1(4), 729-739 (1973)

10. Scheinerman, R., Ullman, D.H.: Fractional Graph Theory-a rational approach to graph theory, http://www.ams.jhu.edu/ ers/fgt (2008)

11. Alpern, S., Gal, S.: The Theory of Search Games and Rendezvous. Springer, Berlin (2003)

12. Alpern, S., Fokkink, R., Kikuta, K.: On Ruckle's conjecture on accumulation games. SIAM J. Control Optim. 48, 5073-5083 (2010)

13. Alpern, S., Fokkink, R.: Accumulation Games on Graphs. LSE-CDAM Research Report Series, vol. 18 (2008)

14. Hoeffding, W., Shrikhande, S.: Bounds for the distribution function of a sum of independent, identically distributed random variables. Ann. Math. Stat. 26(3), 439-449 (1955)

15. Samuels, S.M.: The Markov inequality for sums of independent random variables. Ann. Math. Stat. 40, 1980-1984 (1969)

16. Meester, L.: Extremal distributions for tail probabilities of sums of iid random variables on [0, 1]. In: Proceedings 2008 International Workshop on Applied Probability, Compiegne (IWAP 2008), pp. 1-6 (2008)

17. Hardy, G.H., Wright, E.M.: An Introduction to the Theory of Numbers, 6th revised edn. Oxford University Press, Oxford (2008) 